\title{
Einsatz von Superelementen in der Berechnung von Gittermast-Fahrzeugkranen
}

\author{
DR.-ING. Michael KLEEBERGER, \\ DiPL.-ING. KARL-THOMAS HÜBNER \\ TECHNISCHE UNIVERSITÄT MÜNCHEN, LEHRSTUHL FÜR FÖRDERTECHNIK MATERIALFLUSS UND LOGISTIK
}

In diesem Artikel wird eine Superelementformulierung für geometrisch nichtlineare FiniteElemente-Berechnungen vorgestellt. Die Elementformulierung beruht auf Matrizen, die durch statische Kondensation erzeugt werden. Nach der Festlegung der Elementeigenschaften wird eine Methode zur Berechnung der Elementkräfte bei großen Verschiebungen und Drehungen entwickelt. Damit das Element für die Lösung von Stabilitätsproblemen verwendet werden kann, wird eine Formulierung der nichtlinearen Steifigkeitsmatrix erarbeitet. Eine Beispielanwendung zeigt die Vorteile des Elements bei der Berechnung von Gittermastkranen.

\section{1. $\quad$ Einführung und Problemstellung}

Gittermast-Fahrzeugkrane sind lange, schlanke Systeme mit oft spitzwinkliger Seilabspannung, die im Grenzbereich ein ausgeprägt nichtlineares Last-Verschiebungsverhalten aufweisen. Sie werden in der Praxis vornehmlich durch quasistatische Berechnungen ausgelegt, die in DIN 15018 T3 [DIN84] und DIN EN 13000 [DIN04] spezifiziert sind.

Diese Methode der Auslegung beschreibt die dynamischen Beanspruchungen dieser Krane nur näherungsweise, weshalb die europäischen Normen auch ausdrücklich eine dynamische Auslegungsberechnung erlauben: "Generell können wahlweise fortschrittliche und anerkannte theoretische Methoden (z.B. elastokinetische Analyse zur Simulation der Lasteinwirkungen) ... verwendet werden." [DIN04].

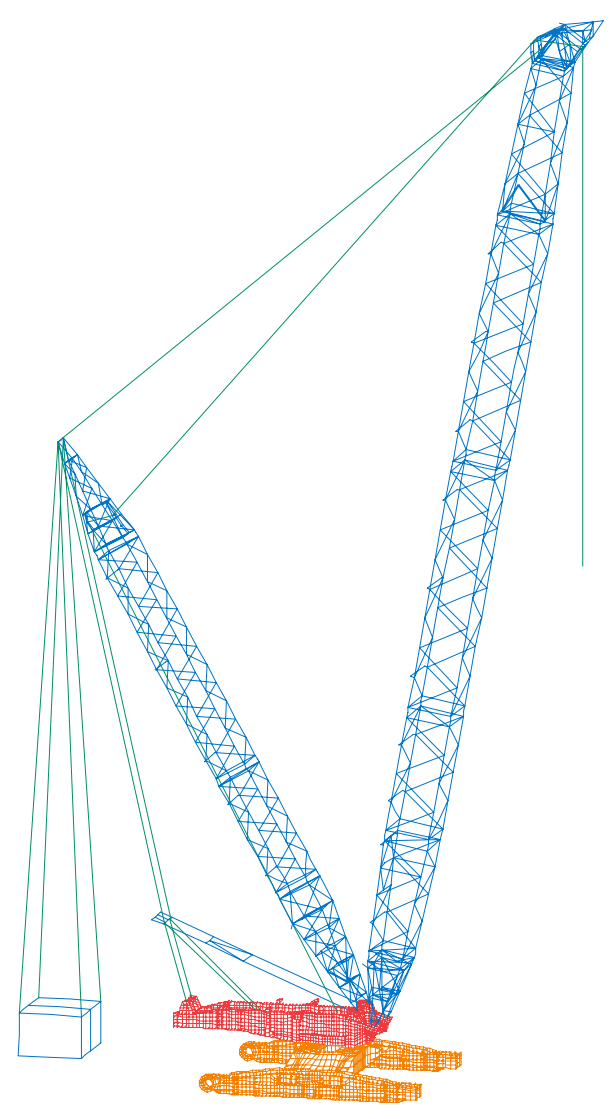

Abbildung 1: Finite-Elemente-Modell eines Gittermast-Fahrzeugkrans 
Abbildung 1 zeigt beispielhaft das Finite-Elemente-Modell eines Gittermast-Fahrzeugkrans. Das Balkenmodell der Gittermastausleger enthält abhängig vom Rüstzustand 200 bis 2000 Knoten. Im Gegensatz zu diesem relativ kleinen Teil des Modells besitzen die Schalenmodelle von Ober- und Unterwagen bis zu 40000 Knoten [Kleeberger96, Günthner97].

Da Gittermast-Fahrzeugkrane nach dem Baukastenprinzip konstruiert sind, lassen sie sich in einer Vielzahl von Rüstzuständen und Auslegerstellungen betreiben. Die Berechnung der Traglasttabellen erfordert bis 1000000 Rechenläufe [Günthner00-1, Günthner00-2, Webhofer05]. Die oben erwähnten Modellgrößen führen dabei zu nicht akzeptablen Rechenzeiten. Daraus entsteht die Notwendigkeit, die Modellgrößen zu reduzieren. In der industriellen Praxis werden die Gittermastbauteile als einzelne Balkenelemente abgebildet. Die Steifigkeiten von Ober- und Unterwagen werden mit Federn berücksichtigt.

Eine genauere Reduktionstechnik ist die statische Kondensation. Sie eignet sich für lineare Systeme, wie die sehr steifen Ober- und Unterwagenmodelle. Dieses Verfahren lässt sich aber nicht direkt zur geometrisch nichtlinearen Berechnung der Auslegermodelle verwenden. Jedoch erfährt jedes Gittermastbauteil für sich alleine betrachtet nur kleine Verschiebungen. Daraus ergibt sich die Zielsetzung, auf der Basis der statischen Kondensation eine Superelementformulierung für geometrisch nichtlineare Finite-Elemente-Berechnungen zu entwickeln.

\section{Statische Kondensation}

Die Grundidee der statischen Kondensation [Bathe96, Szilard82] ist die Reduktion der Knotenanzahl eines Finite-Elemente-Teilmodells durch Elimination der inneren Knoten. Nur Knoten, mit denen das Teilmodell mit anderen Teilmodellen verbunden ist, bleiben erhalten. Abbildung 2 zeigt die Anschlussknoten eines Gittermastzwischenstücks.

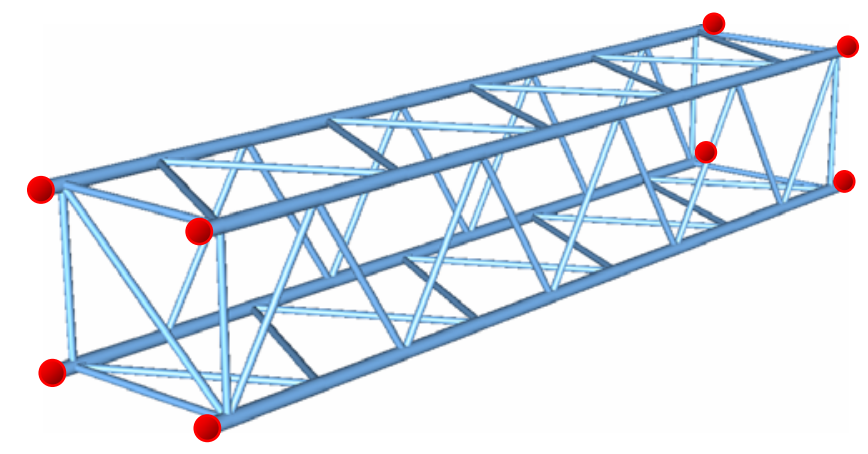

Abbildung 2: Anschlussknoten eines Gittermastzwischenstücks

Die statische Kondensation ist auf lineare Systeme anwendbar. Um die Gleichungen zu entwickeln, werden die Steifigkeitsmatrix und die zugeordneten Verschiebungs- und Lastvektoren folgendermaßen unterteilt:

$$
\left(\begin{array}{c|c}
K_{i i} & K_{i c} \\
---- & ---- \\
K_{c i} & K_{c c}
\end{array}\right) \cdot\left(\begin{array}{c}
u_{i} \\
-- \\
u_{c}
\end{array}\right)=\left(\begin{array}{c}
R_{i} \\
-- \\
R_{c}
\end{array}\right)
$$

Dabei sind $u_{i}$ bzw. $u_{c}$ die Verschiebungsvektoren, die man behalten bzw. herauskondensieren will. Die Matrizen $K_{i i}, K_{i c}$ und $K_{c c}$ und die Vektoren der äußeren Lasten $R_{i}$ und $R_{c}$ entsprechen der Unterteilung der Verschiebungsvektoren $u_{i}$ und $u_{c}$.

Die zweite Matrixgleichung in (1) ergibt:

$$
\underline{u}_{c}=K_{c c}^{-1} \cdot\left(\underline{R}_{c}-K_{c i} \underline{u}_{i}\right)
$$


Setzt man Gleichung (2) für $u_{c}$ in die erste Matrixgleichung in (1) ein, so erhält man die kondensierten Gleichungen

$$
K^{*} \cdot \underline{u}_{i}=\underline{R}_{i}-K_{i c} \cdot K_{c c}^{-1} \cdot \underline{R}_{c}
$$

mit

$$
K^{*}=K_{i i}-K_{i c} \cdot K_{c c}^{-1} \cdot K_{c i}
$$

Die kondensierte Steifigkeitsmatrix $K^{*}$ wird in der Berechnung des Gesamtmodells verwendet.

In nachgeschalteten Rechenläufen lassen sich für alle kondensierten Teilmodelle die Verschiebungsvektoren $u_{c}$ und damit die Elementkräfte und -spannungen berechnen. Bei linearen Systemen entsteht dadurch kein Genauigkeitsverlust.

\section{Nichtlineare Bewegungsgleichung}

Im Gegensatz zu einer linearen Berechnung muss eine nichtlineare Berechnung in inkrementellen Schritten ausgeführt werden. Gleichung (5) beschreibt den Übergang von einem bekannten Zustand zur Zeit $t$ zum Zustand zur Zeit $t+\Delta t$. Im Allgemeinen müssen zur Zeit $t+\Delta t$ Gleichgewichtsiterationen durchgeführt werden [Bathe96].

$$
\begin{gathered}
M \cdot{ }^{t+\Delta t} \underline{\ddot{u}}+D \cdot{ }^{t+\Delta t} \underline{\dot{u}}+{ }^{t} K \cdot \underline{u}={ }^{t+\Delta t} \underline{R}-{ }^{t} \underline{F} \\
{ }^{t+\Delta t} \underline{u}={ }^{t} \underline{u}+\underline{u}
\end{gathered}
$$

mit
M: $\quad$ Massenmatrix
$D: \quad$ Dämpfungsmatrix
${ }^{t} K$ : Tangentensteifigkeitsmatrix zur Zeit $t$
${ }^{t+\Delta t} \underline{R}$ : Vektor der äußeren Lasten zur Zeit $t+\Delta t$
${ }^{t} F$ : Vektor der Elementkräfte zur Zeit $t$
${ }^{t+\Delta t} \underline{u}$ : Vektor der Verschiebungen zur Zeit $t+\Delta t$
${ }^{t} u$ : $\quad$ Vektor der Verschiebungen zur Zeit $t$
$\underline{u}$ : Vektor der inkrementellen Verschiebungen

Die Tangentensteifigkeitsmatrix ${ }^{t} K$ setzt sich aus der linearen Steifigkeitsmatrix ${ }^{t} K_{L}$ und der nichtlinearen Steifigkeitsmatrix ${ }^{t} K_{N L}$ zusammen:

$$
{ }^{t} K={ }^{t} K_{L}+{ }^{t} K_{N L}
$$

Während sich die lineare Steifigkeitsmatrix aus den Materialeigenschaften und Abmessungen des Tragwerks ergibt, berücksichtigt die nichtlineare Steifigkeitsmatrix den Einfluss der Spannungen auf die Systemstabilität.

\section{Superelementformulierung für geometrisch nichtlineare Berechnungen}

Beruhend auf den Matrizen, die durch statische Kondensation erzeugt werden, wird eine Superelementformulierung für statische und dynamische geometrisch nichtlineare Berechnungen entwickelt. Das Element hat folgende grundlegenden Eigenschaften:

- Das Element hat mindestens drei Knoten, die Gesamtanzahl N der Knoten ist unbegrenzt

- Jeder Elementknoten besitzt drei translatorische und drei rotatorische Freiheitsgrade

- Große Verschiebungen und Drehungen sind erlaubt

- Die Verformung des Elements bleibt klein

- Das Element muss sich für Stabilitätsuntersuchungen eignen, d.h. eine Formulierung für die nichtlineare Steifigkeitsmatrix wird entwickelt 


\subsection{Positionierungsproblem}

Abbildung 3 zeigt als Beispiel ein Superelement mit vier Knoten in seinem Anfangszustand zur Zeit 0 und seinem Zustand zur Zeit $t$. Die Koordinaten der Knotenpunkte sind durch den Vektor

$$
\underline{x}=\left(\begin{array}{c}
\underline{x}^{(1)} \\
\vdots \\
\underline{x}^{(N)}
\end{array}\right), \quad \underline{x}^{(i)}=\left(\begin{array}{c}
x \\
y \\
z
\end{array}\right)
$$

bezüglich des globalen Koordinatensystems gegeben.

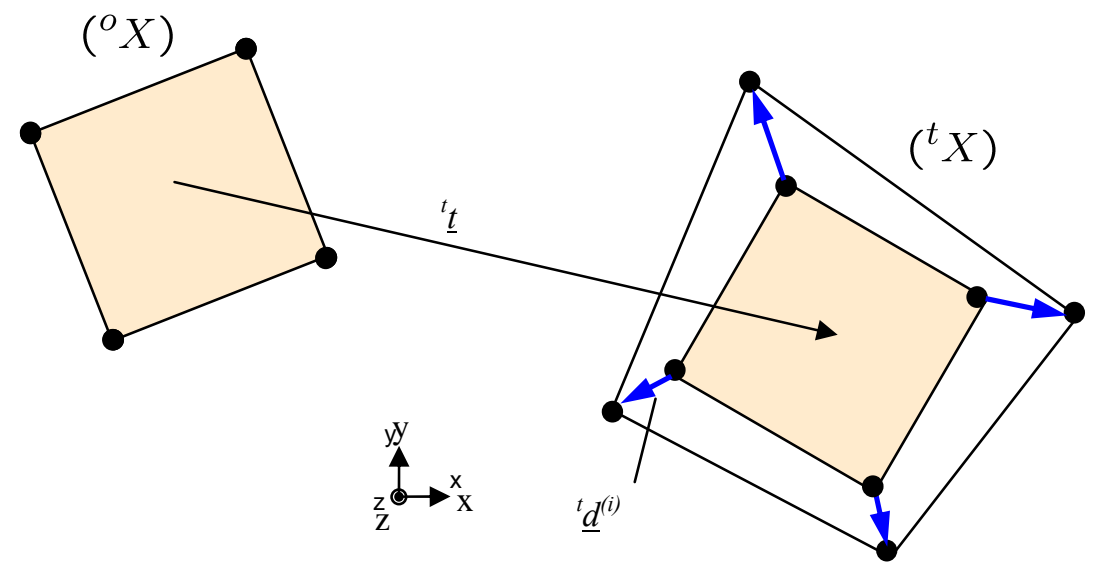

Abbildung 3: Anfangszustand und Zustand zur Zeit $t$

Da das Element große Verschiebungen und Drehungen ausführen kann, müssen die Starrkörperbewegung und die Elementverformung voneinander getrennt werden. Der Ortsvektor zur Zeit $t$ des Knotens $i$ ist gegeben durch

$$
{ }^{t} \underline{x}^{(i)}=\underbrace{{ }^{t} \underline{t}+{ }^{t} T^{T} \cdot{ }^{o} \underline{x}^{(i)}}_{\text {Starrkörperbewegung }}+\underbrace{{ }^{t} \underline{d}^{(i)}}_{\text {Verformung }}
$$

mit

$$
\begin{aligned}
& { }^{t} t: \text { Vektor der Starrkörperverschiebung } \\
& { }^{t} T \text { : Transformationsmatrix }
\end{aligned}
$$

Nach der Starrkörperbewegung sollten die Knoten des unverformten Elements so nahe wie möglich an ihren Positionen zur Zeit $t$ liegen. Ein Weg diese Bedingung zu erfüllen, ist die Minimierung des Verschiebungsvektors ${ }^{t} \underline{d}$, der die Verformung des Elements ausdrückt:

$$
\left|{ }^{t} \underline{d}\right|^{2}=\sum_{i=1}^{N}\left|{ }^{t} \underline{x}^{(i)}-\left({ }^{t} \underline{t}+{ }^{t} T^{T} \cdot{ }^{o} \underline{x}^{(i)}\right)\right|^{2} \rightarrow \text { Min }
$$

Bezieht man ${ }^{t} \underline{x}$ und ${ }^{0} \underline{x}$ auf den Elementschwerpunkt, dann lässt sich Gleichung (9) in folgendes Maximierungsproblem umformen [Nüchter02, Horn87]:

$$
\sum_{i=1}^{N}\left|{ }^{t} \underline{x}^{(i)^{T}} \cdot{ }^{t} T^{T} \cdot{ }^{o} \underline{x}^{(i)}\right|^{2} \rightarrow \operatorname{Max}
$$

Die Transformationsmatrix $T$ wird durch das Einheitsquaternion $q$ ausgedrückt [Blaschke60]:

$$
\begin{gathered}
q_{1}=\cos (\varphi / 2) \\
q_{2}=n_{x} \cdot \sin (\varphi / 2) \\
q_{3}=n_{y} \cdot \sin (\varphi / 2) \\
q_{4}=n_{z} \cdot \sin (\varphi / 2)
\end{gathered}
$$

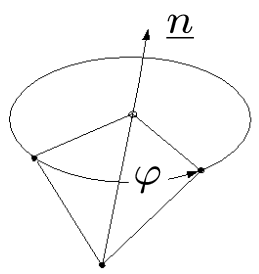




$$
T=\left(\begin{array}{ccc}
q_{1}^{2}+q_{2}^{2}-q_{3}^{2}-q_{4}^{2} & 2\left(q_{2} q_{3}+q_{4} q_{1}\right) & 2\left(q_{2} q_{4}-q_{3} q_{1}\right) \\
2\left(q_{2} q_{3}-q_{4} q_{1}\right) & q_{1}^{2}-q_{2}^{2}+q_{3}^{2}-q_{4}^{2} & 2\left(q_{3} q_{4}+q_{2} q_{1}\right) \\
2\left(q_{2} q_{4}+q_{3} q_{1}\right) & 2\left(q_{3} q_{4}-q_{2} q_{1}\right) & q_{1}^{2}-q_{2}^{2}-q_{3}^{2}+q_{4}^{2}
\end{array}\right)
$$

Mit Hilfe des Quaternions $q$ kann Gleichung (10) wie folgt umgeformt werden

$$
{ }^{t} \underline{q}^{T} \cdot N \cdot{ }^{t} \underline{q} \rightarrow \operatorname{Max}
$$

mit

$$
\begin{aligned}
& N=\left(\begin{array}{cccc}
S_{11}+S_{22}+S_{33} & S_{23}-S_{32} & S_{31}-S_{13} & S_{12}-S_{21} \\
S_{23}-S_{32} & S_{11}-S_{22}-S_{33} & S_{12}+S_{21} & S_{13}+S_{31} \\
S_{31}-S_{13} & S_{12}+S_{21} & S_{22}-S_{11}-S_{33} & S_{23}+S_{32} \\
S_{12}-S_{21} & S_{13}+S_{31} & S_{23}+S_{32} & S_{33}-S_{11}-S_{22}
\end{array}\right) \\
& S_{11}=\sum_{i=1}^{N}\left({ }^{o} x \cdot{ }^{t} x\right)^{(i)} \quad, \quad S_{22}=\sum_{i=1}^{N}\left({ }^{o} y \cdot{ }^{t} y\right)^{(i)} \quad, \quad S_{33}=\sum_{i=1}^{N}\left({ }^{o} z \cdot{ }^{t} z\right)^{(i)} \\
& S_{12}=\sum_{i=1}^{N}\left({ }^{o} x \cdot{ }^{t} y\right)^{(i)} \quad, \quad S_{21}=\sum_{i=1}^{N}\left({ }^{o} y \cdot{ }^{t} x\right)^{(i)} \quad, \quad S_{23}=\sum_{i=1}^{N}\left({ }^{o} y \cdot{ }^{t} z\right)^{(i)} \\
& S_{31}=\sum_{i=1}^{N}\left({ }^{o} z \cdot{ }^{t} x\right)^{(i)} \quad, \quad S_{32}=\sum_{i=1}^{N}\left({ }^{o} z \cdot{ }^{t} y\right)^{(i)} \quad, \quad S_{13}=\sum_{i=1}^{N}\left({ }^{o} x \cdot{ }^{t} z\right)^{(i)}
\end{aligned}
$$

Gemäß [Horn87] ist das gesuchte Quaternion ${ }^{t} q$ der dem größten Eigenwert zugeordnete Eigenvektor des folgenden Eigenwertproblems:

$$
(N-\lambda I) \cdot{ }^{t} \underline{q}=\underline{0}
$$

Aus der Lösung von Gleichung (13) ergibt sich die Transformationsmatrix ${ }^{t} T(q)$. Die Elementverformung im lokalen Koordinatensystem ist durch den Verschiebungsvektor ${ }^{\mathrm{t}} \underline{\underline{d}} \underline{\underline{ }}$ gegeben:

$$
{ }^{t} \underline{d}_{L}^{(i)}={ }^{t} T\left({ }^{t} \underline{q}\right) \cdot{ }^{t} \underline{x}^{(i)}-{ }^{o} \underline{x}^{(i)}
$$

\subsection{Lokale Drehungen}

Zur Berechnung der lokalen Drehungen der Elementknoten wird jedem Knoten ein Quaternion $q_{N}$ zugeordnet. Im Anfangszustand zur Zeit $t=0$ werden alle Quaternionen $q_{N}$ gleich dem Quaternion ${ }^{0} q$ (Orientierung des lokalen Koordinatensystems) gesetzt. Die Quaternionen ${ }^{t} q_{N}$ zur Zeit $t$ ergeben sich aus der Lösung der Bewegungsgleichung (5).

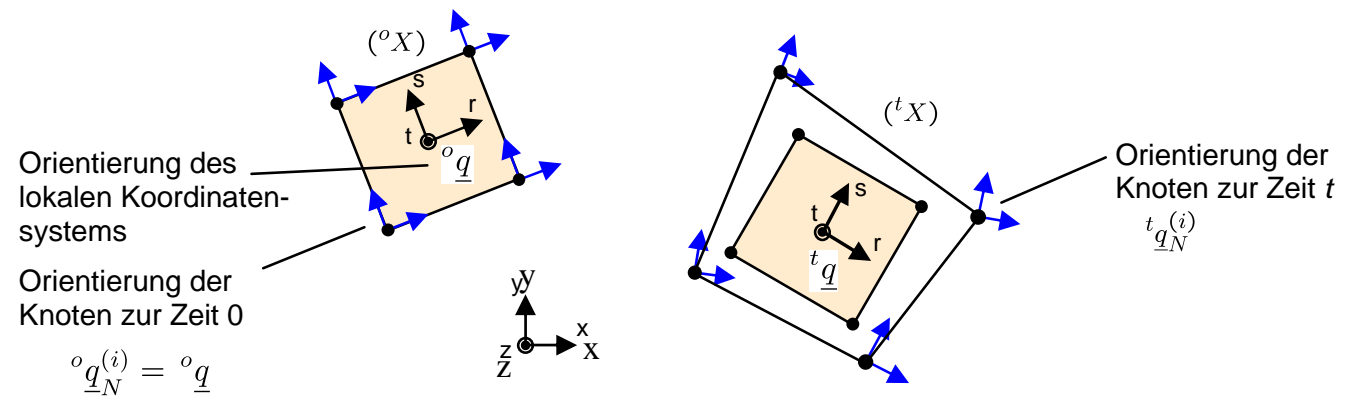

Abbildung 4: Orientierung der Knoten 
Die durch Drehung der Knoten verursachte Elementverformung zur Zeit $t$ ist gegeben durch die Differenz der Drehungen, die durch die Quaternionen ${ }^{t} \underline{q}_{N}$ und dem Quaternion ${ }^{t} \underline{q}$ (Orientierung des lokalen Koordinatensystems zur Zeit $t$ ) beschrieben werden:

$$
\underline{ }^{t} \underline{q i f f}_{\text {Dif }}=\left(\begin{array}{cccc}
{ }^{t} q_{N 1} & -{ }^{t} q_{N 2} & -{ }^{t} q_{N 3} & -{ }^{t} q_{N 4} \\
{ }^{t} q_{N 2} & { }^{t} q_{N 1} & -{ }^{t} q_{N 4} & { }^{t} q_{N 3} \\
{ }^{t} q_{N 3} & { }^{t} q_{N 4} & { }^{t} q_{N 1} & -{ }^{t} q_{N 2} \\
{ }^{t} q_{N 4} & -{ }^{t} q_{N 3} & { }^{t} q_{N 2} & { }^{t} q_{N 1}
\end{array}\right) \cdot\left(\begin{array}{c}
{ }^{t} q_{1} \\
{ }^{t} q_{2} \\
{ }^{t} q_{3} \\
{ }^{t} q_{4}
\end{array}\right)
$$

Unter der Voraussetzung, dass die Elementverformung klein bleibt, kann Gleichung (15) linearisiert werden:

$$
\underline{\varphi}^{t}=\left(\begin{array}{c}
{ }^{t} \varphi_{1} \\
{ }^{t} \varphi_{2} \\
{ }^{t} \varphi_{3}
\end{array}\right)^{(i)} \approx 2 \cdot\left(\begin{array}{c}
{ }^{t} q_{2, \text { Diff }} \\
{ }^{t} q_{3, \text { Diff }} \\
{ }^{t} q_{4, \text { Diff }}
\end{array}\right)^{(i)}
$$

\subsection{Berechnung der Elementkräfte}

Die lokalen Elementkräfte ${ }^{t} \underline{F}_{L}$ hängen von den lokalen Verschiebungen und Drehungen ${ }^{t} \underline{u}_{L}$ und der Steifigkeitsmatrix $K^{*}$ ab:

$$
{ }^{t} \underline{F}_{L}=K^{*} \cdot \underline{u}_{L}
$$

mit

$$
\begin{gathered}
{ }^{t} \underline{u}_{L}=\left(\begin{array}{c}
{ }^{t} \underline{u}_{L}^{(1)} \\
\vdots \\
{ }^{t} \underline{u}_{L}^{(N)}
\end{array}\right) ; \quad{ }^{t} \underline{F}_{L}=\left(\begin{array}{c}
{ }^{t} \underline{F}_{L}^{(1)} \\
\vdots \\
{ }^{t} \underline{F}_{L}^{(N)}
\end{array}\right) \\
{ }^{t} \underline{u}_{L}^{(i)}=\left(\begin{array}{c}
{ }^{t} \underline{d}_{L}^{(i)} \\
--- \\
{ }^{t}{ }^{(i)}
\end{array}\right)=\left(\begin{array}{c}
{ }^{t} T(q) \cdot{ }^{t} \underline{x}^{(i)}-{ }^{o} \underline{x}^{(i)} \\
-------------- \\
{ }^{t} \underline{\varphi}^{(i)}
\end{array}\right)
\end{gathered}
$$

Die globalen Elementkräfte sind

$$
{ }^{t} \underline{F}={ }^{t} \hat{T}^{T} \cdot \underline{F}_{L}
$$

mit

$$
{ }^{t} \hat{T}=\operatorname{diag}\left({ }^{t} T\right)_{2 N}
$$

\subsection{Steifigkeitsmatrix des Superelements}

Die Tangentensteifigkeitsmatrix in Gleichung (5) ist die partielle Ableitung der globalen Elementkräfte ${ }^{t} \underline{F}$ nach den Knotenverschiebungen ${ }^{t} \underline{u}$ [Bathe96]:

$$
{ }^{t} K=\frac{\partial^{t} \underline{F}}{\partial^{t} \underline{u}}
$$

Setzt man die Superelementkräfte aus Gleichung (17) in Gleichung (18) ein, dann ergibt sich:

$$
{ }^{t} K=\underbrace{{ }^{t} \hat{T}^{T} \cdot K^{*} \cdot{ }^{t} \hat{T}}_{K_{L}}+\underbrace{\frac{\partial{ }^{t} \hat{T}^{T}}{\partial^{t} \underline{u}} \cdot{ }^{t} \underline{F}_{L}+\left(\frac{\partial{ }^{t} \hat{T}}{\partial^{t} \underline{u}} \cdot{ }^{t} \underline{F}_{L}\right)^{T}}_{K_{N L}}
$$

Der erste Teil von (20) ist die ins globale Koordinatensystem transformierte Elementsteifig-keitsmatrix $K^{*}$. Der zweite Teil hängt nur von der Lage des Elements und den Elementkräften ab und ist somit die nichtlineare Steifigkeitsmatrix. 
Die Transformationsmatrix ${ }^{t} T$ hängt nicht von den Drehungen, sondern nur von den Verschiebungen der Elementknoten ab. Weil die Änderung von ${ }^{t} \underline{u}$ der Änderung von ${ }^{t} \underline{x}$ entspricht, ergeben sich die partiellen Ableitungen der Elemente von ${ }^{t} T$ (11) zu:

$$
\begin{gathered}
\frac{{ }^{t} T_{11}}{\partial^{t} \underline{u}}=\frac{\partial{ }^{t} T_{11}}{\partial{ }^{t} \underline{x}}=-4\left(q_{4} \cdot \frac{\partial q_{4}}{\partial^{t} \underline{x}}+q_{3} \cdot \frac{\partial q_{3}}{\partial{ }^{t} \underline{x}}\right) \\
\frac{\partial{ }^{t} T_{21}}{\partial^{t} \underline{u}}=\frac{\partial{ }^{t} T_{21}}{\partial^{t} \underline{x}}=2\left(q_{2} \cdot \frac{\partial q_{3}}{\partial^{t} \underline{x}}+q_{3} \cdot \frac{\partial q_{2}}{\partial^{t} \underline{x}}-q_{4} \cdot \frac{\partial q_{1}}{\partial{ }^{t} \underline{x}}-q_{1} \cdot \frac{\partial q_{4}}{\partial^{t} \underline{x}}\right)
\end{gathered}
$$

Die Differentiation von Gleichung (5) unter der Nebenbedingung, dass ${ }^{t} q$ ein Einheitsvektor ist, ergibt:

$$
\frac{\partial^{t} \underline{q}}{\partial^{t} \underline{x}}=\left[\begin{array}{c|c}
(N-\lambda I) & { }^{t} \underline{q} \\
---- & -\underline{-1} \\
{ }^{t} \underline{q}^{T} & 0
\end{array}\right]^{-1}\left[\begin{array}{c}
-\frac{\partial N}{\partial^{t} \underline{x}} \cdot{ }^{t} \underline{q} \\
--- \\
0
\end{array}\right]
$$

Setzt man Gleichung (22) in (21) ein, dann lässt sich die nichtlineare Steifigkeitsmatrix berechnen.

\section{Beispielanwendung}

Die Superelementformulierung wurde in das Finite-Elemente-Programm NODYA [Kleeberger04] implementiert. Abbildung 5 zeigt das in der Beispielanwendung verwendete Modell eines Gittermastkrans. Es besteht aus Unter- und Oberwagen sowie dem Hauptausleger. Zur Modellgenerierung, statischen Kondensation und Auswertung wurde I-DEAS [Unigraphics04] verwendet.

Zum Vergleich der konventionellen Modellierungstechnik mit der Superelementformulierung wurde eine nichtlineare, statische Berechnung des Lastfalls H (Eigenlast, Hublast und Massenkräfte aus Drehen) mit NODYA durchgeführt.

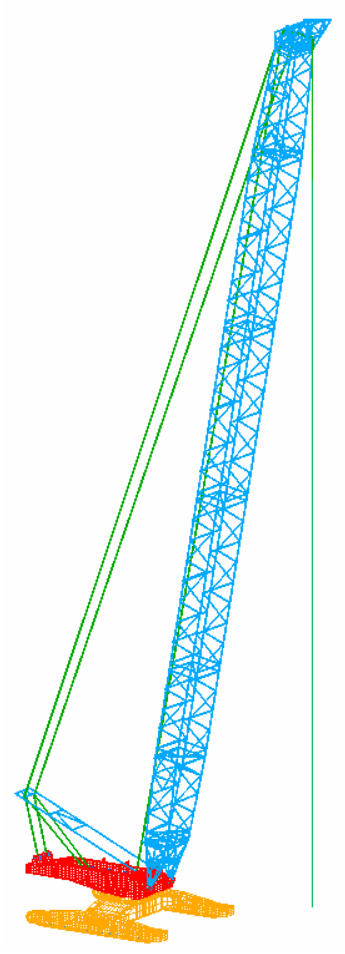

Abbildung 5: Testsystem

Tabelle 1 zeigt die Anzahl der Freiheitsgrade der Originalmodelle und der kondensierten Modelle. Die größte Reduktion erreicht man für den Unter- und Oberwagen. Insgesamt reduziert sich die Anzahl der Freiheitsgrade von 69162 auf 798. 
Tabelle 1: Strukturdaten

\begin{tabular}{|l|l|l|}
\hline & Originalmodell & Superelement \\
\hline Unterwagen & 43740 & 252 \\
\hline Oberwagen & 23028 & 222 \\
\hline Fußstück & 246 & 36 \\
\hline Fußanschlussstück & 222 & 48 \\
\hline Zwischenstück 6m & 288 & 48 \\
\hline Zwischenstück 12m & 402 & 48 \\
\hline Kopfanschlussstück & 222 & 48 \\
\hline Kopfstück & 330 & 60 \\
\hline
\end{tabular}

Es wird ein Vergleich zwischen einem Original-Auslegermodell mit kondensierten Ober- und Unterwagen und einem Gesamtmodell aus Superelementen durchgeführt. Abbildung 6 zeigt die Ergebnisse für zwei verschiedene Hauptauslegerwinkel.

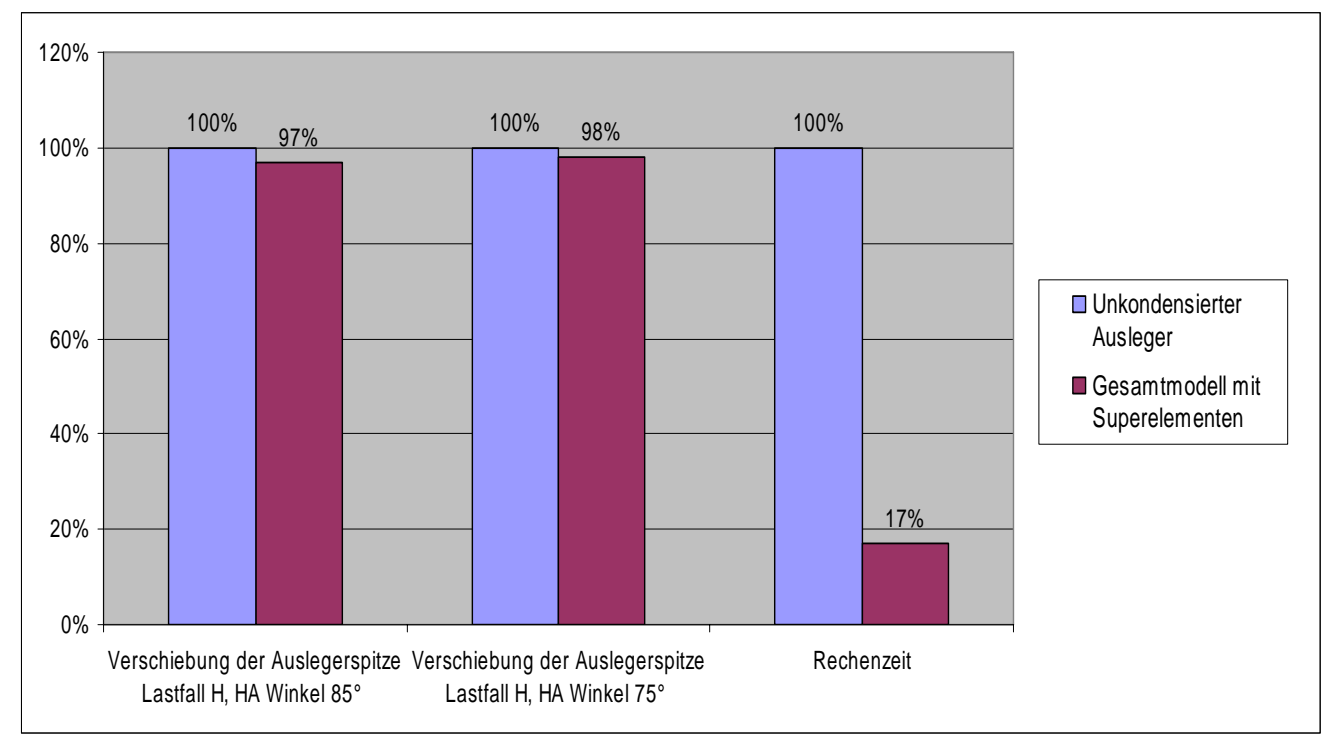

Abbildung 6: Ergebnisse der Beispielanwendung

Die Ergebnisse zeigen einen minimalen Genauigkeitsverlust bei einer signifikanten Reduktion der Rechenzeit.

Weitere Tests zum Verhalten des Superelements in dynamischen Rechenläufen und ein Vergleich mit Mehrkörpersimulationsprogrammen (z.B. [Adams05]) sind in Planung.

\section{Fazit}

Die Berechnung der Traglasttabellen für Gittermastkrane erfordert kleine Finite-Elemente-Modelle. Die in diesem Artikel vorgestellte Superelementformulierung bietet eine hoch entwickelte Reduktionstechnik und eignet sich für geometrisch nichtlineare Berechnungen. Das Element verringert deutlich die Rechenzeit bei einem vernachlässigbaren Genauigkeitsverlust. Die direkte Verknüpfung der kondensierten Modelle mit den Originalmodellen sichert die Konsistenz und erlaubt die Auswertung von Kräften und Spannungen in nachgeschalteten Rechenläufen. 


\section{Literatur}

[Adams05] MSC Software Corporation; MSC.ADAMS 2005 r2 - Installation and Operations Guide : Santa Ana, 2005

[Bathe96] Bathe, K.-J.: Finite Element Procedures : Prentice Hall, 1996

[Blaschke60] Blaschke, W.: Kinematik und Quaternionen. Berlin : VEB Deutscher Verlag der Wissenschaften, 1960

[DIN84] Norm DIN 15018-3:1984-11 : Krane, Grundsätze für Stahltragwerke, Berechnung von Fahrzeugkranen

[DIN04] Norm DIN EN 13000:2004-9 : Krane -Fahrzeugkrane

[Günthner97] Günthner, W. A. ; Kleeberger, M.: Zum Stand der Berechnung von GittermastFahrzeugkranen. In: Deutsche Hebe- und Fördertechnik : dhf 43 (1997) 3, S. 56-61

[Günthner00-1] Günthner, W. A. ; Schröder, F.: Automatisierte Modellgenerierung zur Berechnung von Gittermastkranen - Teil I. In: Fördern und Heben : f+h 50 (2000) 7, S. 506-507

[Günthner00-2] Günthner, W. A. ; Schröder, F.: Automatisierte Modellgenerierung zur Berechnung von Gittermastkranen - Teil II. In: Fördern und Heben : f+h 50 (2000) 8, S. 587-589

[Horn87] Horn, B.: Closed-form solution of absolute orientation using unit quaternions. In: Journal of the Optical Society of America 4 (1987), S. 629-642

[Kleeberger04] Kleeberger, M.: NODYA Version 1.1, Benutzeranweisung. Lehrstuhl für Fördertechnik Materialfluss Logistik, Technische Universität München, 2004

[Kleeberger96] Kleeberger, M.: Nichtlineare dynamische Berechnung von Gittermast-Fahrzeugkranen. München, Technische Universität, Diss., 1996

[Nüchter02] Nüchter, A.: Autonome Exploration und Modellierung von 3D-Umgebungen. Fraunhofer Institut für Autonome Intelligente Systeme, Institut für Informatik III Volltext s. http://www.ais.fraunhofer.de/ARC/3D/scanner/cdindex.html

[Unigraphics04] Unigraphics Solutions Inc.: I-DEAS User's Guide, Cypress, 2004

[Szilard82] Szilard, R.: Finite Berechnungsmethoden der Strukturmechanik, Bd. 1. Berlin u.a.: Verlag von Wilhelm Ernst \& Sohn, 1982

[Webhofer05] Webhofer M.: Verwendung kinematischer Ketten bei der Generierung von Finite-ElementeModellen, In: Logistics Journal : Nicht-referierte Veröffentlichungen, Januar 2005. http://www.elogistics-journal.de/uploads/media/Kinematische_Ketten.pdf, DOI: 10.2195/LJ_Not_Ref_d_Webhofer_0120051 urn:nbn:de:0009-14-7269 